\title{
Formation and Stability of Charged Amino Acid Clusters and the Role of Chirality
}

\author{
B. Concina, P. Hvelplund, A. B. Nielsen, S. Brøndsted Nielsen, \\ and J. Rangama \\ Department of Physics and Astronomy, University of Aarhus, Aarhus, Denmark
}

B. Liu

Physical and Theoretical Chemistry Laboratory, University of Oxford, Oxford, United Kingdom

\section{S. Tomita}

Institute of Applied Physics, University of Tsukuba, Ibaraki, Japan

Amino acid clusters have been studied by several groups and most notably magic number clusters and chiral recognition have been reported. In this work, we have studied the formation of amino acid clusters by electrospray ionization (ESI) and their stability by high-energy collision-induced dissociation (CID). Appearance sizes were determined for multiply charged clusters where the charge is either due to protons or to sodium ions. Finally, we conclude that chiral selectivity plays an important role in cluster formation but seems to be of minor importance for the fragmentation of mixed clusters. (J Am Soc Mass Spectrom 2006, 17, 275-279) (C) 2006 American Society for Mass Spectrometry

I nvestigations of free atomic or molecular clusters have been an active research field for more than two decades. Charged clusters are formed in different types of ion sources [1], and their stability is studied by various techniques including collisions [2], ion-molecule reactions [3], or photon absorption [4]. The clusters investigated are made up of widely different building blocks ranging from atoms [5] over small molecules [6] and $\mathrm{C}_{60}$ [7] to amino acids [8] and even proteins [9].

Conventional cluster sources can be divided into three main groups; supersonic jets, gas aggregation, and surface sources [1]. These cluster sources have been used for production of atomic clusters and clusters of small molecules, while electrospray ionization (ESI) [10] is the preferred method for formation of clusters of biomolecules [8].

The concept of magic numbers that relate to size distributions of clusters in the sense that a cluster containing a magic number of particles shows a particular stability compared with its neighbor clusters has played an important role since the early days of cluster research [11]. Examples of magic numbers are 13, 55, 147 for closed shells of atoms held together by van der Waals forces and 2, 8, 20, 40 for the number of valence electrons in metal clusters. Much of the work related to

Published online January 23, 2006

Address reprint requests to Dr. B. Liu, Physical and Theoretical Chemistry Laboratory, Department of Chemistry, University of Oxford, OX1 3QZ Oxford, United Kingdom. E-mail: bo.liu@chem.ox.ac.uk, and Prof. P. Hvelplund, Department of Physics and Astronomy, University of Aarhus, Ny Munkegade, DK-8000 Aarhus C, Denmark. E-mail: hvelplun@phys.au.dk. biomolecular clusters has focused on clusters of the serine amino acid [8, 12-19]. For these clusters, eight is found to be a magic number and this octamer further shows a preference for homochirality $[8,12]$.

The decay of clusters can follow various routes that depend on cluster conformation, binding forces, activation method, and also charge. Multiply charged clusters can dissociate by charge separation fragmentation "fission" or by evaporating neutral parts [20-22]. In extreme situations, where a cluster is ionized to a high charge state in a short time by radiation from a femtosecond laser, it may undergo a Coulomb explosion resulting in the ejection of many charged fragments [23].

Competition between evaporation and fission in activated multiply charged clusters is another interesting aspect of cluster physics, which can be described by a liquid drop model $[24,25]$. This model has been used for systems as different as atomic nuclei [26] and liquid microdroplets [27]. The limit of stability relates to the smallest size that a droplet can take for a given charge, and likewise the appearance size for a cluster in a given charge state is the smallest number of constituents in the cluster $[6,28]$. This work will focus on clusters of amino acids and collisional activation of these clusters. We will discuss magic numbers and appearance sizes as well as chiral recognition.

\section{Experimental}

The experimental arrangement is described in detail in [29]. The cluster ions were formed by electrospray ionization (ESI). The amino acids were obtained from 


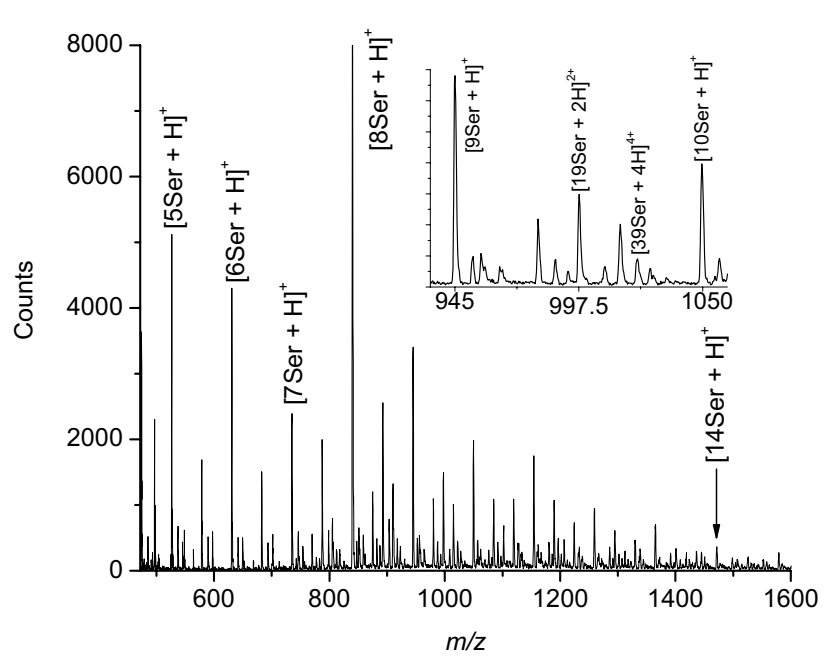

Figure 1. Positive-ion electrospray mass spectrum of an acidic serine solution. The insert focuses on the $\mathrm{m} / \mathrm{z}$ range from [9Ser + $\mathrm{H}]^{+}$to $[10 \text { Ser }+\mathrm{H}]^{+}$and all observed peaks are listed in the text. (Note that some multiply charged multimers with identical $\mathrm{m} / \mathrm{z}$ ratios contribute to the spectrum).

Sigma (Brøndby, Denmark) and dissolved in a water methanol solution $(20 \mu \mathrm{M})$, that was sprayed with a flow rate of $2 \mu \mathrm{l} / \mathrm{min}$. The spray voltage was around 4 $\mathrm{kV}$, the tube lens potential was $60 \mathrm{~V}$, and the heated capillary temperature was set to $60^{\circ} \mathrm{C}$. The ions were accelerated by an electrostatic potential of $50 \mathrm{kV}$. The precursor ions were mass selected with a magnet and passed through a 3-cm long gas cell with entrance and exit apertures of 1 and $3 \mathrm{~mm}$ in diameter containing helium as target gas. The He target gas pressure was monitored by a cold cathode gauge. The product ions exiting the cell were analyzed according to their $\mathrm{m} / \mathrm{z}$ through scanning the voltage of the electrostatic hemispherical analyzer, and fragmentation spectra were obtained. Mass spectra showing the distribution of ions produced in the ion source (magnet scans) were obtained by scanning the magnet with no target gas in the target cell and a fixed analyzer voltage related to the acceleration voltage.

\section{Results and Discussion}

\section{Mass Spectra of Charged Amino Acid Clusters}

A mass spectrum of ions originating from the ESI source when a serine solution is sprayed is shown in Figure 1. The spectrum shows that the serine octamer [8Ser $+\mathrm{H}^{+}$dominates, but singly charged clusters as large as $[14 \mathrm{Ser}+\mathrm{H}]^{+}$are clearly observed. From the insert it is seen that also multiply charged protonated and sodiated clusters are produced in the ESI source. In the interval between the masses of $[9 \mathrm{Ser}+\mathrm{H}]^{+}$and $[10 \mathrm{Ser}+\mathrm{H}]^{+}$the observed peaks can be associated with the following ions, ranked after mass divided by charge: $[27 \mathrm{Ser}+2 \mathrm{H}+\mathrm{Na}]^{3+}(m / z=952),[18 \mathrm{Ser}+\mathrm{H}+$ $\mathrm{Na}]^{2+}(m / z=956),[27 \mathrm{Ser}+\mathrm{H}+2 \mathrm{Na}]^{3+}(m / z=960)$, $[9 \mathrm{Ser}+\mathrm{Na}]^{+}(m / z=968),[28 \mathrm{Ser}+3 \mathrm{H}]^{3+},(m / z=980)$,
$[28 \mathrm{Ser}+2 \mathrm{H}+\mathrm{Na}]^{3+}(m / z=987),[28 \mathrm{Ser}+\mathrm{H}+2 \mathrm{Na}]^{3+}$ $(m / z=994),[19 S e r+2 \mathrm{H}]^{2+}(m / z=998),[19 S e r+\mathrm{H}+$ $\mathrm{Na}]^{2+}(m / z=1008),[29 \mathrm{Ser}+3 \mathrm{H}]^{3+}(m / z=1014),[39 \mathrm{Ser}$ $+4 \mathrm{H}]^{4+}(m / z=1024),[39 \mathrm{Ser}+3 \mathrm{H}+\mathrm{Na}]^{4+}(\mathrm{m} / \mathrm{z}=$ 1029), $[29 \mathrm{Ser}+\mathrm{H}+2 \mathrm{Na}]^{3+}(\mathrm{m} / z=1029)$. A similar spectrum is observed for cysteine clusters. Here the peak corresponding to $[6 \mathrm{Cys}+\mathrm{H}]^{+}$is the most intense and again peaks that can be associated with multiply charged clusters are observed.

\section{Collision Induced Dissociation (CID) of Charged Clusters}

An interesting observation in connection with fragmentation of multiply charged clusters is the so-called appearance size of the cluster $n_{a}$, which refers to the smallest number of constituents observed for a given cluster charge $z$. The simplest model that relates $n_{a}$ to $z$ involves a competition between electrostatic energy and binding energy. Since the total volume of the fragments in a fragmentation process is approximately equal to the initial volume, the decrease in binding energy is compensated by an increase in surface energy. The appearance size is thus determined by a balance between the surface energy and the electrostatic energy. The appearance radius $R_{a}$, which is the smallest radius of a cluster for a given charge state, is thus determined from the relation

$$
\gamma R_{a}^{2} \approx z^{2} e^{2} / R_{a}
$$

where $\gamma$ is the surface tension. Since $n_{a}$ is proportional to $R_{a}{ }^{3}$, this way of reasoning leads to proportionality between $n_{a}$ and $z^{2}$. The same relation was predicted by Lord Rayleigh, [24] who also calculated the constant of proportionality and obtained the relation

$$
R_{a}^{3}=z^{2} e^{2} /(16 \pi \gamma)
$$

This expression predicts $n_{a}$ values which are about a factor of three too small for atomic or molecular clusters [28] but are valid for microdroplets [27].

A mass spectrum with $\mathrm{He}$ as collision gas is shown in Figure 2. The primary beam consists of a mixture of $[11 \mathrm{Ser}+\mathrm{H}]^{+},[22 \mathrm{Ser}+2 \mathrm{H}]^{2+},[33 \mathrm{Ser}+3 \mathrm{H}]^{3+}$, and $[44 \mathrm{Ser}+4 \mathrm{H}]^{4+}$ clusters. The lightest doubly charged ion consists of nine serine molecules and the corresponding number for triply and quadruply charged clusters is 22 and 39 , respectively. In other words, $n_{a}$ is 9 for $z=2,22$ for $z=3$, and 39 for $z=3$. The corresponding numbers for protonated cysteine clusters are 9, 22, and 41. It should be noted from Figure 1 that similar appearance sizes are found from the spectrum resulting from magnet scans which indicate that the way of activation (high-energy collisions versus lowenergy in-source collisions) is not very important for the dissociation mode. In Figure 3, appearance sizes $n_{\mathrm{a}}$ are plotted as a function of cluster charge for several atomic and molecular clusters $[6,30]$. Compared with other 


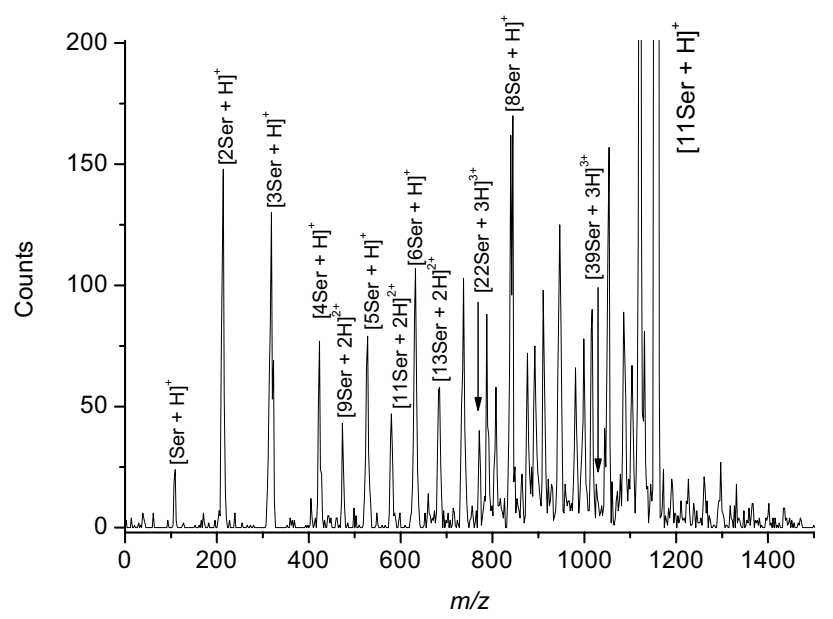

Figure 2. A mass spectrum of serine clusters that have collided with a He gas. The primary beam consists of a mixture of [11Ser + $\mathrm{H}]^{+},[22 \mathrm{Ser}+2 \mathrm{H}]^{2+},[33 \mathrm{Ser}+3 \mathrm{H}]^{3+}$, and $[44 \mathrm{Ser}+4 \mathrm{H}]^{4+}$ clusters. (Note that some multiply charged multimers with identical $\mathrm{m} / \mathrm{z}$ ratios contribute to the spectrum).

clusters of smaller constituents, the appearance sizes $n_{a}$ of amino acid clusters and clusters of $\mathrm{C}_{60}$ are rather small as the size of the constituents and their polarizabilities are large. For serine, a rather large dipole moment also plays a positive role for the stability of the clusters. Taken together, this implies that $n_{a}$ is relatively small for large sticky molecules. It should also be noted that proportionality between $n_{a}$ and $z^{2}$ is obeyed in all cases.

\section{Sodiated and Protonated Clusters}

A mass spectrum originating from collisions between $[15 \mathrm{Ser}+\mathrm{H}+\mathrm{Na}]^{2+}$ and He is shown in Figure 4. A doubly charged odd numbered serine cluster beam was chosen to obtain a "clean" beam. By this choice, singly or triply charged ions can be excluded and, apparently, a possible pollution from quadruply charged ions is

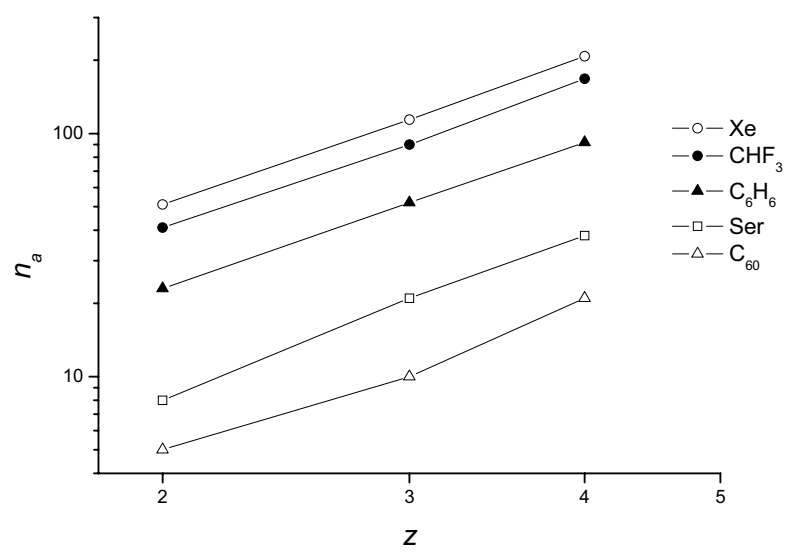

Figure 3. Stability diagram for some atomic and molecular clusters. The points refer to measured appearance sizes $n_{\mathrm{a}}$ for $z$-fold charged clusters. This double-log-plot shows that $n_{\mathrm{a}}$ scales approximately with $z^{2}$.

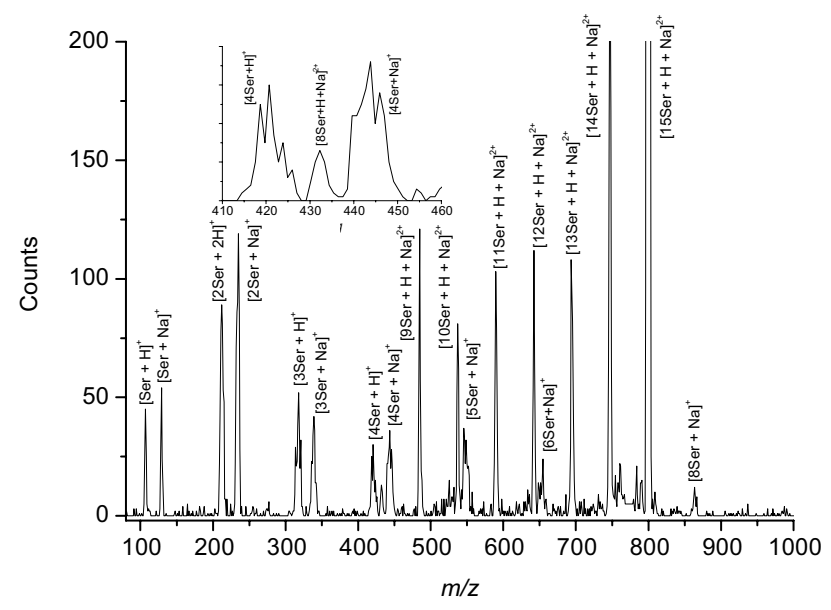

Figure 4. A mass spectrum of $[15 \mathrm{Ser}+\mathrm{H}+\mathrm{Na}]^{2+}$ clusters after collisions with a He gas. The insert highlights singly charged tetramers and the doubly charged octamers.

minute as can be seen from the spectrum. For clusters which are both sodiated and protonated, it is possible to differentiate between singly and doubly charged fragment ions with the same $\mathrm{m} / \mathrm{z}$ value. It is readily seen that the largest singly charged ion is $[8 \mathrm{Ser}+\mathrm{Na}]^{+}$and that the protonated singly charged octamer is missing. The lightest doubly charged ion is $[8 \mathrm{Ser}+\mathrm{H}+\mathrm{Na}]^{2+}$ but the abundance of this ion is small compared with those of the other doubly charged clusters. It should also be noted that the abundance of ions in the interval from $[9 \mathrm{Ser}+\mathrm{H}+\mathrm{Na}]^{2+}$ to $[13 \mathrm{Ser}+\mathrm{H}+\mathrm{Na}]^{2+}$ is relatively large and almost constant while the abundance of $[14 \mathrm{Ser}+\mathrm{H}+\mathrm{Na}]^{2+}$ is a factor of two higher. For the heavy singly charged ions, only the sodiated fragments are observed while for the light fragments protonated and sodiated species appear with similar intensities. Since the proton affinity of serine is five times higher than the sodium affinity [31, 32], it is surprising that peaks related to protonated species are not dominating the spectrum. However, $\mathrm{Na}^{+}$allows for multiple coordination whereas a proton only participates in one hydrogen bond.

As can be seen from the enlarged spectrum shown as an insert in Figure 4, the peaks corresponding to singly charged fragments are considerably broader than the peak assigned to $[8 \mathrm{Ser}+\mathrm{H}+\mathrm{Na}]^{2+}$. From this broadening, the kinetic energy release KER of the dissociation process can be estimated. The KER is between 1 and 3 $\mathrm{eV}$ depending on the primary fragment sizes which corresponds to distances between two point charges from 5 to $15 \AA$ at the moment of fission assuming vacuum between the two charges. These values compare well with the estimated sizes of serine clusters [13].

Based on these observations, we suggest the following decay mechanisms: first, neutral fragments containing from one to six molecules are evaporated until the number of the cluster constituents approaches $n_{a}$. Then, asymmetric fission follow, resulting in singly charged clusters containing one to eight serines succeeded by 

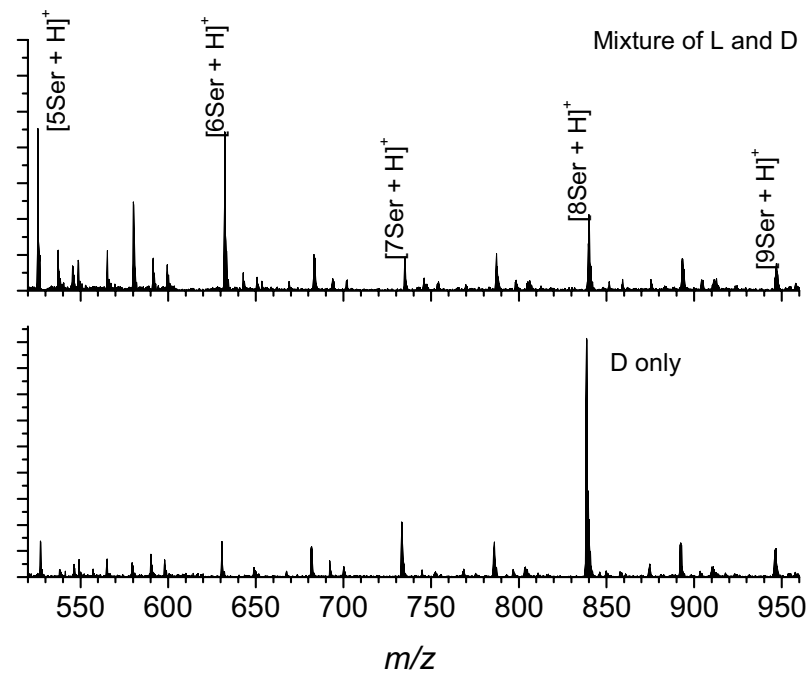

Figure 5. Positive-ion electrospray mass spectrum of an acidic serine solution. The upper spectrum is obtained for a racemic mixture of the L- and D-form and the lower spectrum for the pure D form.

evaporation leading to a distribution of singly charged clusters. The peak broadening is dominated by the fission process, but evaporation prevents a precise measure of the KER.

\section{Homochiral Clusters}

Figure 5 shows data illustrating the role of chirality in serine cluster formation. Spectrum a is obtained with a racemic mixture and Spectrum $b$ with pure L-form serine. The protonated octamer (or multiply charged larger clusters with the same $m / z$ value) is seen to dominate the mass spectrum when pure L-serine is sprayed. When a racemic mixture of $\mathrm{L}$ - and D-serine is sprayed, the spectrum is dominated by peaks at positions with $\mathrm{m} / \mathrm{z}$ values corresponding to $[5 \mathrm{Ser}+\mathrm{H}]^{+}$or $[10 \mathrm{Ser}+2 \mathrm{H}]^{2+}$ and $[6 \mathrm{Ser}+\mathrm{H}]^{+}$or $[12 \mathrm{Ser}+2 \mathrm{H}]^{2+}$. In our experiments clusters with overlapping masses are not separated as they are in the recent work by Julian et al. [14]. These authors found a clear preference for heterochirality for the clusters $[6 \mathrm{Ser}+\mathrm{H}]^{+}$and $[10 \mathrm{Ser}+$ $2 \mathrm{H}]^{2+}$; we therefore ascribe the two prominent peaks in Spectrum a to these two clusters. They also found a strong preference for homochirality for the $[8 \mathrm{Ser}+\mathrm{H}]^{+}$ cluster, again in nice agreement with the present findings.

For mixed clusters, we also found a strong chiral preference. Data were obtained for two solutions containing a one to one mixture of L-serine/L-threonine and L-serine/D-threonine. By assuming that the ion intensities of pure serine octamer in both cases is unity, the ratios of intensities of octamer resulting from these two solutions containing $0,1,2$, or 3 threonine molecules are plotted in Figure 6. In the case of no chiral preference, this ratio should be a constant but it is clearly seen that a serine cluster is less likely to include

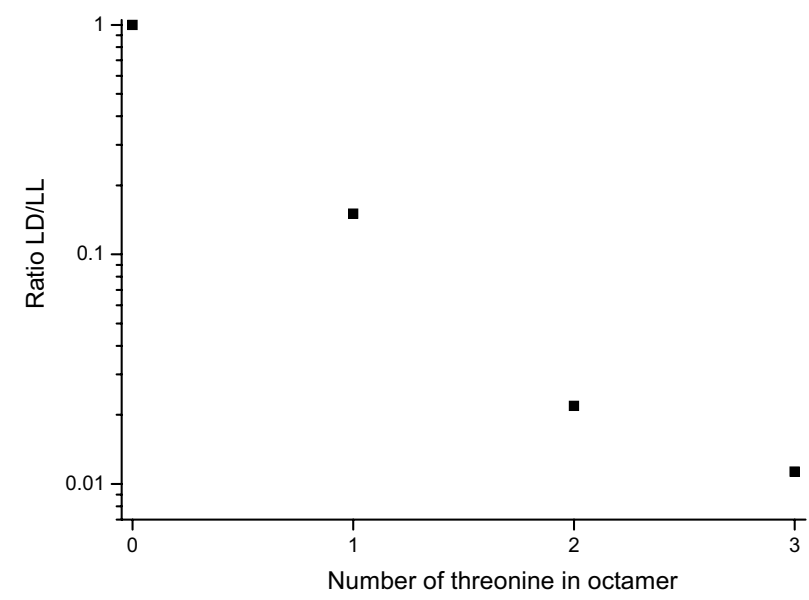

Figure 6. The LD/LL ratio for an octamer consisting of $0,1,2$, or 3 threonine molecules and $8,7,6$, or 5 serine molecules. LL indicates that both molecules are in the $\mathrm{L}$ form in the spray solution while LD refers to the situation where serine is in the $\mathrm{L}$ form and threonine is in the D form.

a threonine molecule with opposite chirality than one with same chirality.

It is, however, still possible to form clusters with say six L-serine and two D- threonine [12]. Figure 7 displays a mass spectrum that shows the CID (helium) fragment distribution for such a cluster beam. The distribution of serine and threonine for a peak with a given number of amino acids is close to a statistical one, but proton affinities also play a role for these distributions. The threonine monomer is, as an example, found to be more abundant than the serine monomer reflecting the larger proton affinity of threonine compared with that of serine [31]. These observations suggest that chiral recognition plays a role in cluster formation but is less important in dissociation.

\section{Conclusions}

Our measurements of cluster formation and destruction have established information on magic numbers, ap-

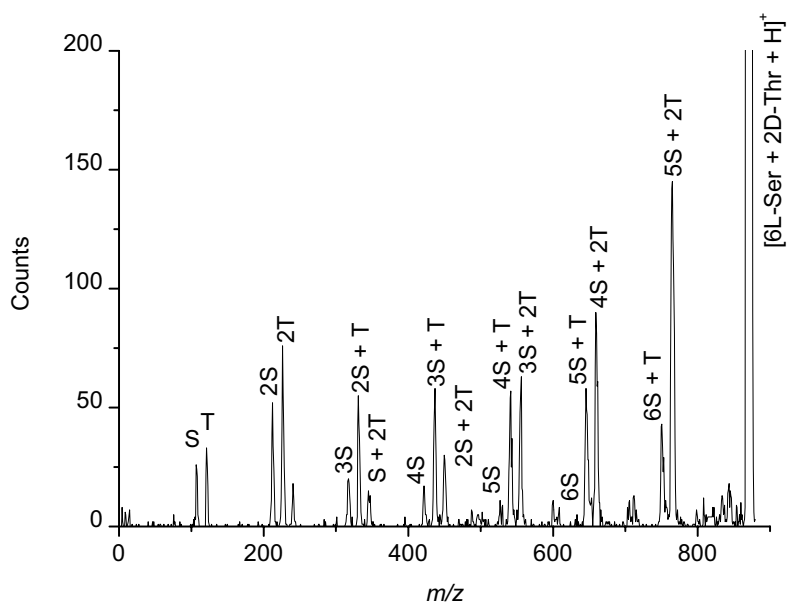

Figure 7. A mass spectrum of a [6L-Ser $+2 \mathrm{D}-\mathrm{Thr}+\mathrm{H}]^{+}$clusters after collisions with a He gas. 
pearance sizes, and chiral recognition for clusters of amino acids. The clusters have been studied by electrospray ionization mass spectrometry and high-energy collisions. Multiply charged clusters dissociate by Coulombically driven fission or by evaporation of neutral amino acids. The smallest size of a cluster with a given charge $z$ is dubbed appearance size $n_{a}$, and it is found that $n_{a}$ is proportional to $z^{2}$ in accordance with theory and experimental observations for clusters of a variety of atomic and molecular building blocks. We have also shown that mixed clusters can reveal chiral recognition in their formation stage but seem to be indifferent to chirality when it comes to dissociation.

\section{Acknowledgments}

This work was supported by the Danish National Research Foundation through the Aarhus Center for Atomic Physics (ACAP) and by the EU Research Training Network, contract HPRN-CT-2000-0002. SBN acknowledges support from the Danish Natural Science Research Council.

\section{References}

1. Haberland, H. In Clusters of Atoms and Molecules I; Haberland, H., Ed.; Springer Verlag: Berlin, Germany, 1994; p 207.

2. Jarrold, M. F.; Bower, J. E. Collision Induced Dissociation of Aluminum Cluster Ions with Chemisorbed Oxygen, $\mathrm{Al}_{n} \mathrm{O}_{\mathrm{m}}^{+}(n=3-26, m=1,2)$ : Influence of Electronic Structure on Stability. J. Chem. Phys. 1987, 87, 1610-1619.

3. Mandich, M. L.; Bondybey, V. E.; Reents, W. D., Jr. Reactive Etching of Positive and Negative Silicon Cluster Ions by Nitrogen Dioxide. J. Chem. Phys. 1987, 86, 4245-4257.

4. Liu, Y.; Zhang, Q.-L.; Tittel, F. K.; Curl, R. F.; Smalley, R. E. Photodetachment and Photofragmentation Studies of Semiconductor Cluster Anions. J. Chem. Phys. 1986, 85, 7434-7441.

5. Frauendorf, S. G.; Guet, C. Atomic Clusters as a Branch of Nuclear Physics. Annu. Rev. Nucl. Part. Sci. 2001, 51, 219-259.

6. Echt, O.; Kreisle, D.; Recknagel, E.; Saenz, J. J.; Casero, R.; Soler, J. M. Dissociation Channels of Multiply Charged van der Waals Clusters. Phys. Rev. A 1988, 38, 3236-3248.

7. Martin, T. P.; Naher, U.; Schaber, H.; Zimmermann, U. Clusters of Fullerene Molecules. Phys. Rev. Lett. 1993, 70, 3079-3082.

8. Cooks, R. G.; Zhang, D.; Koch, K. J.; Gozzo, F. C.; Eberlin, M. N. Chiroselective Self-Directed Octamerization of Serine: Implications for Homochirogenesis. Anal. Chem. 2001, 73, 3646-3655.

9. Jurchen, J. C.; Garcia, D. E.; Williams, E. R. Gas-Phase Dissociation Pathways of Multiply Charged Peptide Clusters. J. Am. Soc. Mass Spectrom. 2003, 14, 1373-1386.

10. Fenn, J. B.; Mann, M.; Meng, C. K.; Wong, S. F.; Whitehouse, C. M. Electrospray Ionization for Mass Spectrometry of Large Biomolecules. Science 1989, 246, 64-71.
11. Knight, W. D.; Clemenger, K.; de Heer, W. A.; Saunders, W. A.; Chou, M. Y.; Cohen, M. L. Electronic Shell Structure and Abundances of Sodium Clusters. Phys. Rev. Lett. 1984, 52, 2141-2144.

12. Julian, R. R. Hodyss, R. Kinnear, B. Jarrold, M. F · Beauchamp, J. L. Nanocrystalline Aggregation of Serine Detected by Electrospray Ionization Mass Spectrometry: Origin of the Stable Homochiral Gas-Phase Serine Octamer. J. Phys. Chem. B. 2002, 106, 1219-1228.

13. Counterman, A. E.; Clemmer, D. E. Magic Number Clusters of Serine in the Gas Phase. J. Phys. Chem. B 2001, 105, 8092-8096.

14. Julian, R. R.; Myung, S.; Clemmer, D. E. Spontaneous Anti-Resolution in Heterochiral Clusters of Serine. J. Am. Chem. Soc. 2004, 126, 4110-4111.

15. Koch, K. J.; Gozzo, F. C.; Zhang, D.; Eberlin, M. N.; Cooks, R. G. Serine Octamer Metaclusters: Formation, Structure, Elucidation, and Implications for Homochiral Polymerization. Chem. Commun. 2001, 1854-1855.

16. Koch, K. J.; Aggerholm, T.; Nanita, S. C.; Cooks, R. G. Clustering of Nucleobases with Alkali Metals Studied by Electrospray Ionization Tandem Mass Spectrometry: Implications for Mechanisms of Multistrand DNA Stabilization. I. Mass Spectrom. 2002, 37, 676-686.

17. Takats, Z.; Nanita, S. C.; Cooks, R. G. Serine Octamer Reactions: Indicators of Prebiotic Relevance.Angew. Chem. Int. Ed. 2003, 42, 35213523 .

18. Schalley, A.; Weis, P. Unusually Stable Magic Number Clusters of Serine with a Surprising Preference for Homochirality. Int. J. Mass Spectrom. 2002, 221, 9-19.

19. Koch, K. J.; Gozzo, F. C.; Nanita, S. C.; Takats, Z.; Eberlin, M. N.; Cooks, R. G. Chiral Transmission Between Amino Acids: Chirally Selective Amino Acid Substitution in the Serine Octamer as a Possible Step in Homochirogenesis. Angew. Chem. Int. Ed. 2002, 41, 1721-1724.

20. Brechignac, C.; Cahuzac, P.; Carlier, F.; de Frutos, M. Asymmetric Fission of $\mathrm{Na}^{++}{ }_{n}$ Around the Critical Size of Stability. Phys. Rev. Lett. 1990, 64, 2893-2896.

21. Brechignac, C.; Cahuzac, P.; Carlier, F.; de Frutos, M.; Leygnier, J.; Roux, J. P. Coulombic Fission and Evaporation of Antimony Cluster Ions. J. Chem. Phys. 1995, 102, 763-769.

22. Krückeberg, S.; Dietrich, G.; Lützenkirchen, K.; Schweikhard, L. Walther, C.; Ziegler, J. Fission Barriers of Doubly Charged Silver Clusters. Eur. Phys. 1999, 145-148.

23. Kumarappan, V.; Krishnamurthy, M.; Mathur, D. Asymmetric HighEnergy Ion Emission from Argon Clusters in Intense Laser Fields. Phys. Rev. Lett. 2001, 87, 085005.

24. Strutt, J. W. On the Equilibrium of Liquid Conducting Masses Charged with Electricity. Philos. Mag. 1882, 14, 185-186.

25. Naher, U.; Bjørnholm, S.; Frauendorf, S.; Garcias, F.; Guet, C. Fission of Metal Clusters. Phys. Rep. 1997, 285, 245-320.

26. Bohr, N.; Wheeler, J. A. The Mechanism of Nuclear Fission. Phys. Rev. 1939, 56, 426-450.

27. Duft, D.; Lebius, H.; Huber, B. A.; Guet, C.; Leisner, T. Shape Oscillations and Stability of Charged Microdroplets. Phys. Rev. Lett. 2002, 89, 084503.

28. Chandezon, F.; Tomita, S.; Cormier, D.; Grübling, P.; Guet, C.; Lebius, H.; Pesnelle, A.; Huber, B. A. Rayleigh Instabilities in Multiply Charged Sodium Clusters. Phys. Rev. Lett. 2001, 87, 153402.

29. Boltalina, O. V.; Hvelplund, P.; Jørgensen, T. J. D.; Larsen, M. C.; Larsson, M. O.; Sharoitchenko, A. Electron Capture by Fluorinated Fullerene Anions in Collisions with Xe Atoms. Phys. Rev. A 2000, 62, 23202.

30. Manil, B.; Maunoury, L.; Huber, B. A.; Jensen, J.; Schmidt, H. T.; Zettergren, H.; Cederquist, H.; Tomita, S.; Hvelplund, P. Highly Charged Clusters of Fullerenes: Charge Mobility and Appearance Sizes. Phys. Rev. Lett. 2003, 91, 215504.

31. Hunter, E. P.; Lias, S. G. Evaluated Gas Phase Basicities and Proton Affinities of Molecules: An Update. J. Phys. Chem. Ref. Data 1998, 27, 413-656.

32. Gapeev, A.; Dunbar, R. C. $\mathrm{Na}^{+}$Affinities of Gas-Phase Amino Acids by Ligand Exchange Equilibrium. Int. J. Mass Spectrom. 2003, 228, 825-839. 